\title{
Enfermedad de Castleman. Análisis histopatológico e inmunohistoquímico de treinta y nueve casos
}

\author{
Diana Brisa Sevilla-Lizcano¹, Christian Lizette Frias-Soria y Carlos Ortiz-Hidalgo ${ }^{1,2}$ \\ ${ }^{1}$ Departamento de Patología Quirúrgica y Molecular, Centro Médico ABC; ${ }^{2}$ Departamento de Biología Celular y Tisular. Universidad Panamericana, \\ Ciudad de México, México
}

\begin{abstract}
Resumen
Introducción: La enfermedad de Castleman (EC) es poco frecuente y se distinguen dos subtipos clínicos (unicéntrica y multicéntrica) y dos patrones histológicos básicos (tipo hialino-vascular [HV]) y células plasmáticas [CP]). Algunos casos de EC multicéntrica están asociados con infección por virus del herpes humano 8 (HHV-8). Objetivos: Presentar las características histopatológicas e inmunohistoquímicas (IHQ) de 39 casos de EC. Método: Se revisaron casos de EC de los archivos del departamento de patología del Centro Médico ABC de la Ciudad de México. Hubo 39 casos de EC y en nueve de ellos se realizaron estudios de IHQ complementaria con desmina, citoqueratina OSCAR (CO) y el receptor del factor de crecimiento epidérmico (EGFR) para evaluar la población de células dendríticas. Resultados y conclusiones: De los 39 casos de EC, 24 fueron HV y 15 CP. Todos los casos HV fueron unicéntricos y solo uno de los casos de CP fue multicéntrico. La localización más frecuente en ambos subtipos fue en los ganglios linfáticos, con 21 de los 24 casos en EC-HV y en los 15 casos de EC-CP. A todos los casos se les realizó CD20 con expresión predominante en los centros germinales (CG), CD3 en la zona paracortical y CD21 en células dendríticas foliculares (CDF) de los CG con expansión hacia la zona del manto hiperplásica (solo en la variante HV). Uno de los casos de EC-CP fue positivo a HHV-8. De los nueve casos (seis HV y tres $C P$ ) en que se realizaron estudios complementarios de IHQ hubo expresión de EGFR en las CDF en ocho y expresión de desmina en ocho en las células reticulares fibroblásticas. La CO fue positiva en tres de seis de los casos de la variante HV y en todos los casos de CP. Las características clínicas, histopatológicas y la presencia de virus de la inmunodeficiencia humana y de HHV-8 permiten la clasificación de la EC en grupos con diferente evolución clínica.
\end{abstract}

PALABRAS CLAVE: Enfermedad de Castleman hialino-vascular. Enfermedad de Castleman multicéntrica. Células reticulares fibroblásticas citoqueratina positivas. Receptor del factor de crecimiento epidérmico. Desmina.
Abstract
Introduction: Castleman disease $(C D)$ is a rare lymphoproliferative that comprises two distinct clinical subtypes (unicentric and multicentric) and has two basic histopathology patterns that are hyaline-vascular (HV) and plasma-cell (PC) type. Some cases of multicentric PC disease are associated with HHV-8 infection. Objective: To present the histopathologic and immunohisto- chemical characteristics of 39 cases of $C D$. Methods: A review of cases with the diagnosis $C D$ from the files of the Department of Pathology of the ABC Medical Centre in Mexico City was performed. Thirty-nine cases of CD were identified, and a detailed paraffin immunophenotypic study of 9 of them was completed using desmin, cytokeratin OSCAR (CO) and Epidermal growth factor receptor (EGFR), to evaluate the dendritic cell population. Results and Conclusions: Of the 39 cases of CD, 24 were HV and 15 CP. All HV cases were unicentric and only one case of CP was multicentric. The most frequent localization in both subtypes was in lymph nodes; 21/24 cases in HV and 15 cases of CP. All cases were immunostained with CD20 that was

\author{
Correspondencia: \\ Carlos Ortiz-Hidalgo \\ Departamento de Biología Celular y Tisular \\ Escuela de Medicina \\ Universidad Panamericana \\ Donatello, 59 \\ Col. Insurgentes Mixcoac \\ C.P. 03720, Ciudad de México, México \\ E-mail: ortizhidalgocarlos@gmail.com
}
Fecha de recepción: 04-07-2017
Fecha de aceptación: 05-07-2017
DOI:10.24875/GMM.17003021
Gac Med Mex. 2017;153:550-558
Contents available at PubMed www.gacetamedicademexico.com 
expressed in the germinal centers (CGs), CD3 in the paracortical zone, and CD21 in follicular dendritic cells (CDF) within CGs, with expansion towards the area of the hyperplastic mantle zone (only in the HV variant). One case of $C D C P$ was positive for $H H V-8$. Of the nine cases (6 HV and 3 PC cases) that were detailed with IHC, we found EGFR expression in FDC in all but one of the 9 cases studied and desmin was positive in fibroblastic reticulum cells (FRC) in all, but one of the cases of CD. CO was positive FRC in 3 of 6 cases of HV type and all (3) of the PC type. Clinical, histopathological and HIV and HHV-8 status markers, allow for the classification of $C D$ into groups with markedly different outcomes and disease associations.

KEY WORDS: Castleman's disease hyaline vascular type. Castleman's disease plasma cell variant. Cytokeratin positive fibroblastic reticulum cells. Desmin. Epidermal growth factor receptor.

\section{Introducción}

La enfermedad de Castleman (EC) es un grupo heterogéneo de trastornos linfoproliferativos no neoplásicos divididos en la variante hialino-vascular (HV) y la variante de células plasmáticas (CP), ambas con histología y curso clínico variables ${ }^{1-3}$. La forma unicéntrica (localizada) de ambas tiene buen pronóstico, mientras que la forma multicéntrica (sistémica), que comúnmente ocurre en un escenario de pacientes inmunocomprometidos, tiene una alta morbilidad y mortalidad con pocas opciones terapéuticas ${ }^{1,4,5}$. Aunque no se considera una lesión neoplásica, la variante de CP multicéntrica es tratada como tal y está asociada a riesgo incrementado de desarrollar linfoma difuso de células grandes B y sarcoma de Kaposi ${ }^{3,5,6}$.

La EC HV frecuentemente es unicéntrica ${ }^{7} y$, aunque poco frecuente, se puede asociar con la aparición de sarcomas de células dendríticas foliculares de manera sincrónica o metacrónica ${ }^{8,9}$.

Hay informes de la expresión del receptor del factor de crecimiento epidérmico (EGFR, por sus siglas en inglés) en las CDF de ambas variedades, y se ha propuesto una posible relación patogénica ${ }^{2,8,9}$. Además, existe un aumento de CDF (CD21 positivas) y de células reticulares fibroblásticas (CRF) (desmina y citoqueratina positivas) en ambas variantes de $\mathrm{EC}$, sin poder precisar su función o importancia diagnóstica ${ }^{8,10-13}$.

El objetivo de este manuscrito es presentar el estudio clinicopatológico e inmunohistoquímico de 39 casos de EC analizados en una misma institución. Además, en nueve de estos casos se complementó el estudio inmunohistoquímico y se valoró la expresión de EGFR dentro de los centros germinales, y la distribución de las CDF y CRF en ambos subtipos de la EC.

\section{Métodos}

Del archivo del Departamento de Patología Quirúrgica y Molecular del Centro Médico ABC (CMABC) se buscaron los casos con diagnóstico de EC en un periodo de 15 años (2000 a 2015) y se encontraron 39 casos, de los se obtuvieron datos como sexo, edad y localización, y la hoja de diagnóstico histopatológico. De los 39 casos, 30 (76.92\%) correspondieron a materiales externos recibidos para consulta de diagnóstico histopatológico y nueve a pacientes que llegaron al CMABC por adenomegalias.

Para el diagnóstico de EC-HV, los criterios histológicos fueron la presencia de folículos linfoides atróficos con áreas variables de hialinización, zonas del manto expandidas en anillos concéntricos (apariencia en «telas de cebolla»), expansión paracortical con incremento de vénulas de endotelio alto y ausencia parcial o completa de senos subcapsulares.

Para el diagnóstico de EC-CP los criterios histológicos fueron la presencia variable de folículos linfoides atróficos o hiperplásicos, con capa del manto variable y expansión de la paracorteza con numerosas CP policlonales.

A los 39 casos se les determinó para diagnóstico CD20 (BioSB/L26/1:500), CD3 (BIOCARE/M/1:50), CD138 (BioSB/EP201/1:80), Kappa (BioSB/L1C1/1:400), Lambda (BioSB/lambda14/1:400), CD21 (Dako/1F8/1:30) y HHV-8 (human herpesvirus 8, BioSB/13B10/1:10).

En nueve casos se llevó a cabo la detección, por inmunohistoquímica con desmina (BioSB/EP15/1:20), citoqueratina OSCAR (BioSB/OSCAR/1:30) y EGFR (BioSB/31G7/1:100) para valoración de la expresión de estos antígenos en las CDF y las CRF.

\section{Resultados}

De los 39 casos de EC, 24 (62\%) fueron HV y $15(38 \%)$ fueron CP. De los 24 casos de EC-HV, $16(67 \%)$ fueron mujeres y $8(33 \%)$ fueron hombres, con un rango de edad de 3-64 años (promedio de 31 años); en cinco pacientes no contamos con la edad. De los 15 casos de EC-CP, cuatro fueron mujeres (26.5\%) y 11 fueron hombres (73.5\%), y tuvieron un rango de edad de 12-74 años (con un promedio de 46 
años); en cinco pacientes no contamos con la edad). Todos los casos de EC-HV fueron unicéntricos y solo uno de los casos de EC-CP fue multicéntrico.

La localización más frecuente en los 24 casos de EC-HV fue en los ganglios linfáticos (21 casos: $87.5 \%$ ): dos en los ganglios linfáticos axilares, cuatro en los ganglios linfáticos cervicales, dos en los ganglios linfáticos peripancreáticos, dos en los ganglios linfáticos de mediastino, uno en un ganglio linfático de la región perirrenal, uno en un ganglio linfático de la región suprarrenal, uno en un ganglio yugulo-gástrico y otro en un ganglio linfático de la región retroperitoneal. Un caso se presentó en tejidos blandos del hombro, y dos en el pulmón. Los siete casos restantes fueron en ganglios linfáticos, pero no estaba especificada su localización en la hoja de envío.

Histológicamente, la EC-HV presentaba folículos linfoides hiperplásicos redondos o irregulares, de tamaño similar dispersos en la corteza y la médula del ganglio linfático (Fig. 1 A). Algunos de estos folículos presentaban dos o más centros germinales referidos como «centros germinales gemelos» (twinning) (Fig. 1 B). Los centros germinales eran atróficos y muchos de ellos con fibrosis y escasos linfocitos pequeños y numerosas CDF dentro de las áreas fibrosas (Fig. 1 C). En la paracorteza se identificaron numerosas vénulas de endotelio alto con fibrosis leve perivascular, y en forma dispersa estos vasos penetraban radialmente hacia los centros germinales formando estructuras que semejan a una paleta en espiral (Iollipop) (Fig. 1 B y C). En algunos casos, dentro de los centros germinales

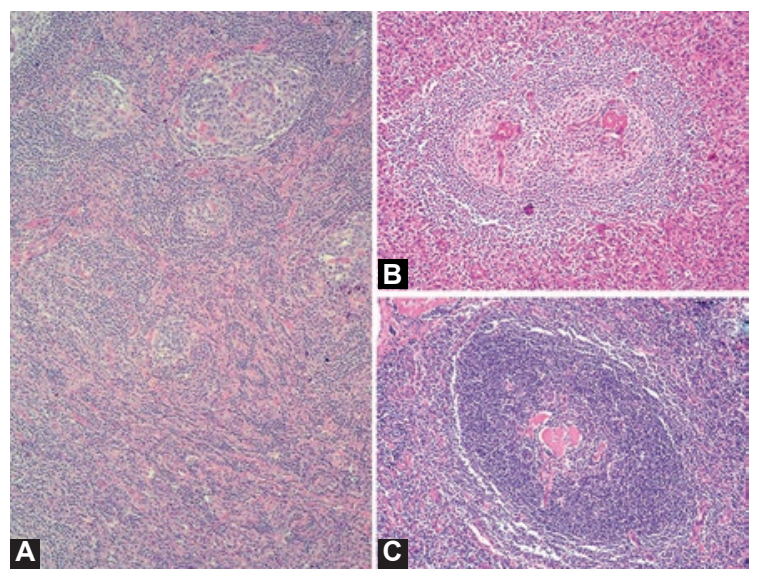

Figura 1. Enfermedad de Castleman variante HV. A: centros germinales, algunos de ellos atróficos, y proliferación vascular paracortical. B: folículos linfoides con dos o más centros germinales referidos como "Centros germinales gemelos" (twinning): algunos vasos de la paracorteza penetran radialmente hacia los centros germinales, formando lesiones que semejan a una paleta en espiral (Iollipop). C: centro germinal con fibrosis central, con formación de anillos concéntricos, con linfocitos pequeños, que le daba un aspecto de "telas de cebolla» (onion skin). atróficos había CDF displásicas con núcleos grandes de contornos irregulares, con cromatina granular y nucléolos pequeños (Fig. $2 \mathrm{~A}$ a C). Estas células dendríticas se extendían hacia la zona de células del manto hiperplásica (Fig. 2 C). Las zonas del manto de estos folículos eran amplias y formaban anillos concéntricos de linfocitos pequeños, que les daban un aspecto de «telas de cebolla» (onion skin). Había además en la paracorteza escasas CP, inmunoblastos, linfocitos pequeños y eosinófilos. Los mantos de CP en la paracorteza, como lo observado en la EC-CP, estaban ausentes. En la mayoría de los casos de la variante HV, los sinusoides subcapsulares de los ganglios linfáticos estaban obliterados. Se identificó esclerosis densa de distribución multifocal en algunos ganglios linfáticos.

Con relación a la EC-CP, la localización del total de los 15 casos fue: cuatro en ganglio linfático cervical, tres en ganglios linfáticos axilares, uno en ganglio linfático supraclavicular, uno en ganglio linfático inguinal, uno en ganglio linfático mesentérico, uno en ganglio linfático cístico y cuatro en ganglios linfáticos sin referir la localización.

Histológicamente, los casos de EC-CP mostraban folículos linfoides normales 0 atróficos con centros gemínales con polaridad conservada y variable zona del manto, y característicamente numerosas CP en las áreas interfolicular y medular con formas binucleadas ocasionales (Fig. $3 \mathrm{~A}$ a C). La proliferación vascular en las áreas interfoliculares fue variable. Los sinusoides ganglionares estuvieron presentes, algunos de ellos ligeramente distendidos. Las zonas del manto estaban bien definidas entre las áreas de paracorteza, pero no había hiperplasia como en la EC-HV. En un caso hubo células más grandes de aspecto plasmablástico que predominaban en la zona del manto y dentro de la paracorteza. Estas células son las que fueron positivas por inmunohistoquímica para el HHV8 (virus asociado a sarcoma de Kaposi) (vide infra).

Los 39 casos fueron positivos al CD20, con la expresión predominante en los centros germinales. Los linfocitos $\mathrm{T}$ fueron positivos para CD3 predominantemente en la zona paracortical. El CD21 mostró una distribución constante de CDF dentro de los centros germinales con positividad intensa y con expansión hacia la zona del manto hiperplásica en la variante HV (Fig. 4). En los 15 casos de la variante CP se identificaron áreas de centros germinales positivos al CD20 y zonas paracorticales expandidas positivas al CD3. El CD21, en estos casos, no mostró expansión hacia la capa del manto. En las zonas paracorticales se identificaron numerosas CP positivas al CD138, todas ellas policlonales. 


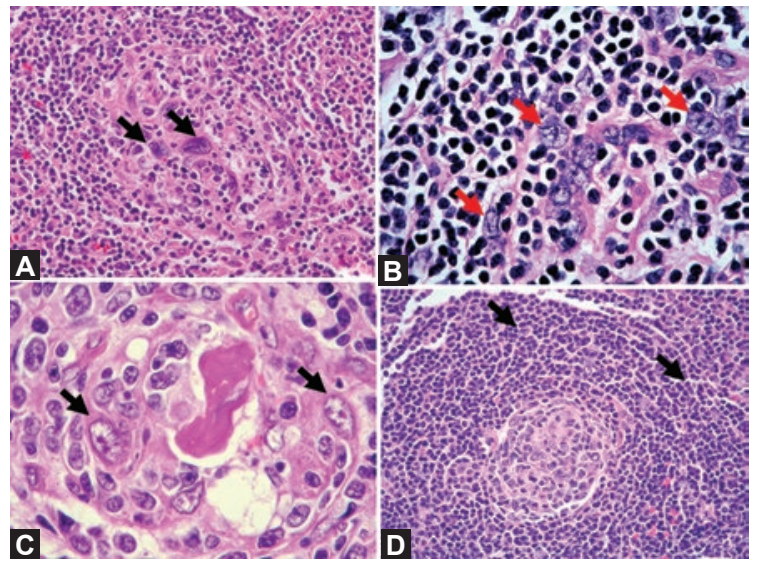

Figura 2. A, B y C: los centros germinales atróficos de la variante HV contienen aumento de CDF, algunas de ellas con datos de displasia (flechas) caracterizada por agrandamiento nuclear con cromatina granular y nucléolos visibles. D: las CDF migran hacia la zona del manto (flechas).

A los 39 casos se les realizó inmunomarcación con HHV-8 y todos los casos de EC-HV fueron negativos; solo un caso de los 15 de la variante CP (que fue el multicéntrico) fue positivo en plasmablastos localizados en la zona del manto y en la región paracortical.

De los nueve casos con los que contamos con bloques de parafina se realizaron estudios complementarios de inmunohistoquímica con desmina y citoqueratina OSCAR. Seis casos (67\%) fueron EC-HV y tres casos (33\%) fueron EC-CP. De los seis casos de EC-HV, cinco fueron mujeres y uno fue un hombre, con un rango de edad de 19 a 55 años (con un promedio de 36 años). De los tres casos de EC-CP, uno fue mujer y dos fueron hombres, con un promedio de edad de 55 años (40-56 años).

La expresión de desmina evaluó la presencia de CRF. En la variante HV encontramos positividad citoplásmica difusa en tres casos, multifocal en un caso y focal en un caso, y resultó negativo en un caso (Fig. 5 A). En la variante CP hubo positividad difusa en un caso y positividad multifocal en dos casos (Fig. 5 B). Morfológicamente, en ambas variantes, estas células eran delgadas, con prolongaciones digitiformes largas distribuidas tanto en la periferia de la zona del manto de los folículos como en la zona paracortical, alrededor de algunas vénulas de endotelio alto y de distribución subcapsular (Fig. 5 A y B).

Con la citoqueratina OSCAR se evaluó igualmente la presencia de CRF. En la variante HV hubo positividad multifocal en dos de los 6 casos (Fig. 5 C), y en la variante CP hubo positividad multifocal en los tres casos. Estas células presentaron morfología digitiforme con prolongaciones largas distribuidas predominantemente en la paracorteza y alrededor de las vénulas del endotelio alto (Fig. $5 \mathrm{C}$ y D).
La expresión de EGFR se observó tanto en el citoplasma como en las membranas de las CDF de los centros germinales, y tanto en la variante HV (Fig. 6 A) como en la CP (Fig. 6 B). La expresión de EGFR fue proporcional a la displasia que mostraban las CDF, es decir, cuanto mayor fue la expresión de EGFR, mayor fue la displasia en las CDF (Fig. 6 A y B).

\section{Discusión}

La EC, también conocida como hiperplasia angiofolicular, hiperplasia ganglionar gigante o hamartoma angiomatoso linfoide, es una linfadenopatía reactiva poco frecuente ${ }^{3,13,14}$. Fue descrita por primera vez en 1954 por Benjamin Castleman (1906-1982) en un paciente varón de 40 años de edad quien se presentó con fiebre alta, sudoración, fatiga y tos no productiva, con una masa en el mediastino anterior ${ }^{15}$. Los diagnósticos clínicos presuntivos fueron teratoma, tuberculoma, timoma y linfoma de Hodgkin ${ }^{3,15}$. Histológicamente, Castleman identificó una forma peculiar de hiperplasia linfoide caracterizada por formación de centros germinales atróficos con fibrosis hialina variable, lo que remedaba estructuras similares a los cuerpos de Hassall en el timo, pero sin queratina central. Además, en la parte de la periferia de los centros germinales observó un patrón arremolinado de linfocitos de la zona del manto que semejaba «telas de cebolla», y en la zona paracortical había proliferación vascular acentuada ${ }^{10,15}$. Dos años más tarde, Castleman informó nuevamente este caso junto con 12 más, los cuales eran tumores mediastinales que habían sido diagnosticados previamente como timomas $^{16}$. Con esto realizó la descripción de una nueva enfermedad que indicó que no era neoplásica ni de origen tímico, que corresponde a lo que hoy conocemos como la variante HV y lleva el nombre de enfermedad de Castleman ${ }^{3,6,15,16}$. En 1969, Fledring y Schillings describieron la variedad $\mathrm{CP}$, que fue invariablemente asociada con la presentación clínica con fiebre, linfadenopatía, esplenomegalia y anemia $^{17}$, y en 1972 Keller, Hochholzer y Castleman definieron y separaron las variantes HV y CP en una revisión de 81 casos $^{18}$. En 1983, Frizzera, et al. ${ }^{19}$ informaron las características clínicas, morfológicas y de inmunofenotipo de 15 pacientes con EC de afección multicéntrica (ECM), en la que clínicamente todos los pacientes tenían fiebre, sudoración nocturna y pérdida de peso. Morfológicamente, los ganglios afectados tenían arquitectura conservada con expansión paracortical con plasmocitosis difusa marcada y con algunos centros germinales prominentes con cambios vasculares hialinizados focales, es decir, 


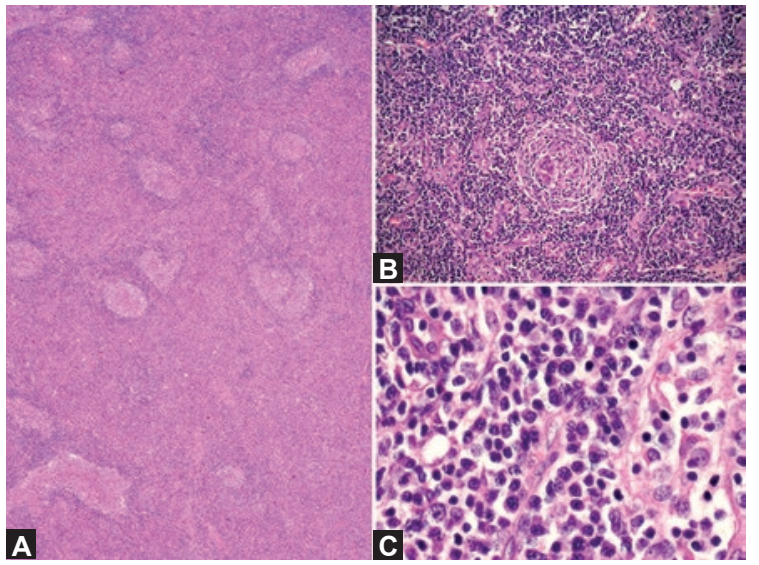

Figura 3. A: la variante $C P$ muestra folículos linfoides grandes o de tamaño normal, con centros gemínales con polaridad conservada. B y C: hay numerosas células plasmáticas en las áreas interfolicular y medular, con formas binucleadas ocasionales.

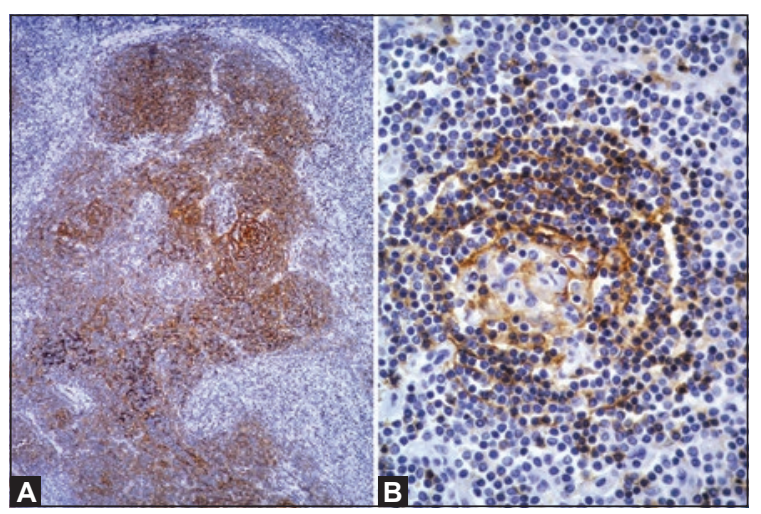

Figura 4. Con el CD21 las CDF mostraron una distribución constante dentro de los centros germinales, con positividad intensa y con expansión acentuada hacia la zona del manto hiperplásico en la variante HV (A), más delimitada al centro germinal y solo focalmente hacia la zona del manto en la variante $\mathrm{CP}(\mathbf{B})$.

presentaban características tanto de la variante HV como de la variante $\mathrm{CP}^{19,20}$. Algunos pacientes infectados con el virus de la inmunodeficiencia humana $(\mathrm{VIH})$ presentan ECM variante $\mathrm{CP}$, y fueron Soulier, et al. ${ }^{21}$ quienes identificaron el HHV-8 en todos los pacientes con ECM VIH positivos y en el $40 \%$ de los $\mathrm{VIH}$ negativos.

La EC es un grupo de enfermedades poco frecuentes cuya prevalencia no está bien establecida, pero se calcula en aproximadamente un caso por 100000 habitantes en los EE.UU.. Afecta predominantemente los ganglios linfáticos de tórax, cuello y abdomen, en un 70 , un 15 y un $15 \%$, respectivamente, aunque se han descrito casos en pulmón, laringe, parótida, páncreas y meninges ${ }^{22}$. Clínicamente puede estar localizada en un ganglio o un conglomerado ganglionar (unicéntrica), o puede afectar a dos o más ganglios 0 conglomerados ganglionares (multicéntrica) ${ }^{2}$.
Histológicamente se clasifica en variante HV y variante $\mathrm{CP}$, pero ocasionalmente se han informado $\mathrm{EC}$ con características mixtas ${ }^{3,23}$. Sin embargo, como la mayoría de los casos de la variante CP pueden presentar algunas características de la variante HV, muchos autores consideran estos casos como mixtos dentro del espectro de la variante $\mathrm{CP}^{24}$. La clasificación actual de la EC comprende: 1) la variante HV; 2) la variante $C P$ unicéntrica; 3 ) la variante $C P$ multicéntrica asociada a HHV-8; y 4) la variable CP multicéntrica idiopática (ECMi) ${ }^{20}$ (Fig. 7).

La variante HV representa el $90 \%$ de los casos de EC y corresponde a la mayoría de los casos de EC unicéntrica ${ }^{3,4}$. Existen pocos informes de casos de ECHV multicéntrica, y estos posiblemente correspondan a la variante $\mathrm{CP}^{6,9}$. La EC-HV se presenta en un rango amplio de edad, pero ocurre con mayor frecuencia en adultos jóvenes con una edad media de diagnóstico en la tercera o la cuarta década de la vida. No tiene predilección por ningún sexo y la mayoría de los pacientes presentan una masa localizada sin síntomas constitucionales ni anormalidades del laboratorio. El mediastino es la localización más común, seguido de los ganglios linfáticos del cuello y del abdomen ${ }^{20}$.

Se han sugerido numerosos factores etiológicos para su desarrollo, como fenómenos autoinmunitarios, inmunodeficiencia, inflamación crónica de bajo grado, respuesta a una causa ambiental desconocida e infección por el virus de Epstein-Barr (VEB). Sin embargo, su causa aún es desconocida1.

Histológicamente, los centros germinales suelen ser son atróficos, con escasos linfocitos, y contienen una proporción aumentada de CDF, algunas de ellas con datos de displasia, caracterizada por un agrandamiento nuclear con cromatina granular y nucléolos visibles. Los linfocitos de la zona del manto se disponen en anillos concéntricos dando un aspecto de patrón en «telas de cebolla». Los folículos a menudo son penetrados radialmente por vasos sanguíneos, procedentes de la zona paracortical, dando la apariencia de paleta en espiral (lollipop) ${ }^{5}$. Cada folículo puede contener uno o más centros germinales ${ }^{10,25}$, y la región interfolicular muestra numerosas vénulas de endotelio alto².

La EC-HV, de acuerdo con Keller, et al. ${ }^{10,25}$, puede presentar dos variedades: 1) el «tipo folicular», que presenta numerosos folículos linfoides en más del $50 \%$ de la superficie del ganglio linfático afectado, con alteraciones del centro germinal que incluyen lisis folicular, transformación progresiva de centros germinales, hiperplasia del células del manto y centros germinales atróficos (kokima); y 2) el subtipo «rico en 


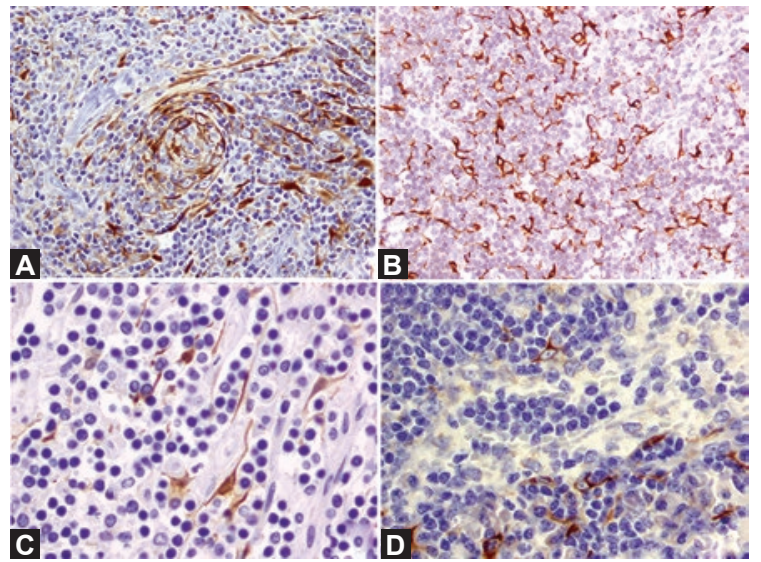

Figura 5. Las células reticulares fibroblásticas fueron positivas a desmina tanto en la variante $H V(\mathrm{~A})$ como en la variante $\mathrm{CP}(\mathrm{B})$. Además, estas células expresaron citoqueratina OSCAR tanto en la variante $H V$ (C) como en la variante CP (D). En ambos casos, y con ambas inmunomarcaciones, la distribución de estas células fue predominantemente en la zona paracortical.

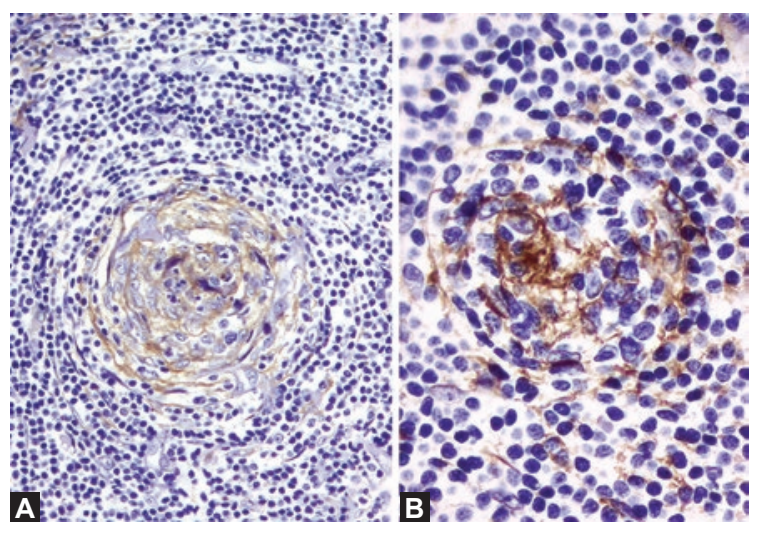

Figura 6. La expresión de EGFR se observó tanto en el citoplasma como en las membranas de las CDF de los centros germinales, tanto en la variedad $H V(\mathbf{A})$ como en la variedad $C P(\mathbf{B})$.

estroma», que está caracterizado por áreas extensas de expansión paracortical (interfoliculares) con una red compleja de vénulas de endotelio alto, y estas áreas deben corresponder a más del $50 \%$ de la superficie del ganglio linfático afectado $0^{5,10,25}$.

Las CDF de los centros germinales pueden presentar datos variables de displasia, y han sido descritos sarcomas de CDF asociados a la EC ${ }^{2,8,9,26}$. De hecho, estudios moleculares recientes han demostrado clonalidad en las CDF de la variante HV y sugieren que la alteración genética en estas células pueden ser la causa de la enfermedad ${ }^{26-28}$. Es de interés que las CDF en los casos de EC, tanto HV como CP, presentan expresión difusa e intensa de EGFR, que no se observa en las CDF de otras enfermedades, como diversos linfomas y ganglios reactivos, dato que puede ser de utilidad en el diagnóstico diferencial con otros procesos reactivos ${ }^{8,9,26-28}$. De nuestros nueve casos en los que inmunomarcamos con EGFR, ocho resultaron positivos en intensidad variable en las CDF.

Normalmente, en la zona interfolicular de los ganglios linfáticos hay CRF que representan el $20-50 \%$ del compartimento no hematopoyético ${ }^{12,13}$. Estas células fueron descritas en 1987 por Franke y Moll ${ }^{29}$, y subsecuentemente confirmadas por Coggi, et al..$^{30}$, y presentan citoplasma alargado que se encuentra en contacto con otras células similares y crean una red tridimensional en la que los leucocitos migran y transportan antígenos solubles y algunas moléculas de señalización en los ganglios linfáticos ${ }^{31,32}$. Estas CRF proporcionan parte de la estructura ganglionar, contribuyen a la compartimentalización de las células $B$ y las células $T$, y dirigen el tráfico de leucocitos a través de la secreción de quimiocinas (IL-7 y CCL-9) implicadas en la supervivencia de los linfocitos $T^{31,32}$. Estas CFR son positivas a desmina, actina y algunos tipos de citoqueratina, y se encuentran localizadas en la paracorteza, predominantemente distribuidas alrededor de las vénulas del endotelio alto, y en la región subcapsular ${ }^{10,12,30-32}$. Existen pocos datos sobre el papel fisiopatológico de estas células en el diagnóstico de la $\mathrm{EC}^{10}$. Danon, et al..$^{10}$ han informado la presencia de estas células como parte de la proliferación de la variante rica en estroma, sin poder precisar su exacta función. Nosotros las encontramos en todas las variedades, expresando tanto desmina como citoqueratina en diferentes proporciones.

El diagnóstico diferencial de la variante HV puede abarcar diversas condiciones. Algunos ganglios linfáticos pudieran presentar cambios focales semejantes a la EC, que han sido llamados «cambios castlemanoides", y pueden presentarse en diversas condiciones reactivas o neoplásicas, como en el linfoma de Hodgkin o diversos linfomas no Hodgkin, en particular el linfoma de células del manto ${ }^{33-35}$. También se han informado cambios "castlemanoides» en la linfadenopatía por VIH, en particular en los cambios histológicos descritos en el "patrón C", que representa la etapa llamada de agotamiento (burnout stage) de la infección. Hay que hacer énfasis en que estos cambios son focales y no generalizados como en la $\mathrm{EC}^{3,33}$.

El timoma es otra condición que entra en el diagnóstico diferencial de la variante HV de la EC. De hecho, como ya fue expuesto, en su descripción original Castleman indicó que la enfermedad que él estaba describiendo semejaba al timoma. Tanto el timoma como la variante HV pueden presentarse en el mediastino, y los centros germinales atróficos hialinizados de la EC-HV pueden semejar a los corpúsculos de Hassall; sin embargo, hay que recordar que 
la presencia de corpúsculos de Hassal es muy rara en el timoma ${ }^{5,25}$

La variante de CP unicéntrica (CPU) representa menos del $10-20 \%$ de los casos de EC y se presenta en una población de pacientes similar a la variante HV, pero con predominio en hombres y algunos de ellos inmunocomprometidos ${ }^{1,20,33}$. En muchos de los casos informados como EC variante CPU, al hacer estudios de extensión se han encontrado otros ganglios linfáticos afectados, por lo que más bien corresponden a la variedad multicéntrica ${ }^{3,4,6}$. La EC-CPU se asocia frecuentemente con manifestaciones sistémicas, como fiebre, sudoración nocturna, pérdida de peso, malestar general, alteraciones hematológicas tales como anemia, trombocitopenia e hipergammaglobulinemia y esplenomegalia ${ }^{1,3,7}$. En estos pacientes hay un incremento de la interleucina 6 humana (IL-6h) sérica, que es a lo que se atribuye la linfadenopatía y las manifestaciones sistémicas de la enfermedad (vide infra) ${ }^{1,7,33}$.

El HHV-8 desempeña un papel predominante en la patogénesis de la variante $\mathrm{CP}^{1,23,36}$. El HHV-8 es un rhadinovirus descubierto en 1994 por Chang, et al. ${ }^{37}$ como "virus herpes gamma» en el sarcoma de Kaposi de un paciente con sida, y tiene una prevalencia estimada del $25 \%$ en los EE.UU. $23,24,37$. Como otros virus tipo herpes, el HHV-8 tiene dos fases principales de expresión genética: una fase latente y una lítica. En la fase latente hay liberación de genes de latencia, como el antígeno nuclear de latencia, y en la fase lítica es donde ocurre la producción de nuevos viriones y predomina la liberación de IL-6 viral (IL-6v) ${ }^{6,23}$.

El ciclo lítico del HHV-8 en las células linfovasculares del ganglio linfático conduce a la destrucción de estas células, lo que conlleva la formación de una cicatriz hialina, mientras que la producción de IL-6v provoca neoangiogénesis a través del factor de crecimiento de fibroblastos y del factor de crecimiento del endotelio vascular ${ }^{3,23}$. Esta angiogénesis está representada en las numerosas vénulas del endotelio alto presentes tanto en la paracorteza ganglionar como en los vasos penetrantes al centro germinal de los pacientes con EC-CPU.

Además, el incremento en la producción de IL-6h conduce a la proliferación de plasmablastos, que se localizan predominantemente alrededor de las vénulas del endotelio alto. Si estos plasmablastos se encuentran en pequeños agregados, entonces se considera que son microlinfomas ${ }^{1,3,9,38}$ que se definen como una proliferación monoclonal de plasmablastos que expresan $\lg \lambda$ y HHV-8 ${ }^{20,38}$. Se ha propuesto que el proceso fisiopatológico principal de la EC-CP es la hiperproliferación de los linfocitos $B$ provocada por señales paracrinas y autocrinas de la IL-6v y de la IL-6h, $\mathrm{h}^{3,6,23}$.

La EC-CP se clasifica de acuerdo con la expresión de HHV-8, el cual puede ser positivo o negativo ${ }^{36}$ (Fig. 7). La variante CP HHV-8 positivo generalmente tiene un curso más agresivo que su contraparte HHV-8 negativo ${ }^{33}$. La infección por HHV-8 se detecta hasta en el $50 \%$ de los casos de EC-CP y hasta en el $100 \%$ de los pacientes infectados con $\mathrm{VIH}^{3,23,24}$. Se ha planteado la hipótesis de que la asociación de EC y sarcoma de Kaposi en un solo ganglio linfático se debe a la infección lítica por HHV-8 del endotelio linfovascular y de los linfocitos $B^{3,23}$.

Histológicamente, la EC-CPU puede tener folículos linfoides «normales» o presentar hiperplasia variable de centros germinales con expansión inconstante de la

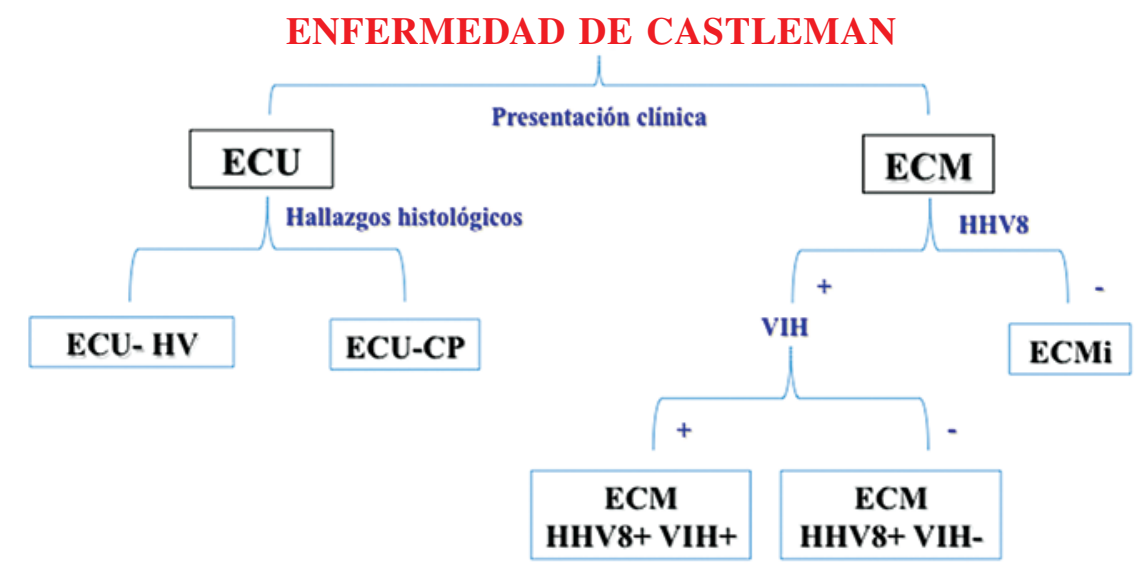

Figura 7. Clasificación actual de la enfermedad de Castleman 20. CP: células plasmáticas; ECM: enfermedad de Castleman multicéntrica; ECMi: enfermedad de Castleman multicéntrica idiopática; ECU: enfermedad de Castleman unicéntrica; HHV: virus de herpes humano; HV: hialino-vascular; VIH: virus de la inmunodeficiencia humana. 
zona del manto. Algunos de estos folículos pueden tener cambios similares a los observados en la variante HV, es decir, con atrofia de centros germinales, fibrosis variable e hipervascularizados; sin embargo, estos cambios pueden no estar presentes. Una característica de la variante $\mathrm{CP}$ es la expansión interfolicular a expensas de numerosas CP. En aproximadamente el $50 \%$ de los casos estas $\mathrm{CP}$ pueden expresar restricción a cadenas ligeras, predominantemente lambda ( $\lg G \lambda / \lg A \lambda)$, y en el otro $50 \%$ las CP son politípicas ${ }^{3,38}$.

El diagnóstico diferencial de la EC-CP debe ser con enfermedades que se caracterizan por presentar hiperplasia folicular marcada y plasmocitosis interfolicular, como la linfadenopatía en pacientes con artritis reumatoide, el linfoma linfoplasmacítico/macroglobulinemia de Waldenström, el plasmocitoma, el linfoma angioinmunoblástico y la linfadenitis por VIH patrón $\mathrm{C}^{3,33}$.

En 1978 se publicó el primer caso de variante multicéntrica HHV-8+ de la EC, por Gaba, et al. ${ }^{39}$, quienes la separaron de la forma unicéntrica por su naturaleza multifocal ${ }^{39}$. Esta variante es una enfermedad poco frecuente cuya incidencia y prevalencia son inciertas, con patogénesis diferente y con un pronóstico mucho peor que el de otras variantes de la $\mathrm{EC}^{6}$. En el año 2010, Soulier, et al. ${ }^{21}$ describieron la ECM HHV-8+, que puede o no estar asociada al $\mathrm{VIH}$. Virtualmente, todos los casos de ECM en pacientes $\mathrm{VIH}$ positivos están asociados con infección por HHV-8, pero la ECM con HHV-8 también puede ocurrir en pacientes VIH negativos (Fig. 7)1,6,33.

La ECM CP ocurre en un grupo de mayor edad (edad media en la sexta década de la vida). Se presenta en pacientes inmunodeprimidos y en pacientes VIH positivos, y se manifiesta comúnmente con linfadenopatía generalizada, síntomas constitucionales y alteraciones hematológicas con valores séricos de IL-6 elevados durante los episodios sintomáticos ${ }^{6}$. Los pacientes típicamente se presentan con fiebre, sudoraciones, fatiga, caquexia, linfadenopatía, esplenomegalia, citopenia e hipoalbuminemia, síntomas y signos a menudo graves que pueden conducir a la muerte del paciente ${ }^{6}$.

La patogénesis de la ECM HHV-8+ se atribuye a la proliferación de linfocitos $B$ infectados por HHV-8 que producen IL-6v y desregulan la IL-6h $\mathrm{h}^{6,38}$. El exceso de producción de IL-6 es una propiedad compartida con la enfermedad de Crohn y la artritis reumatoide, que estimula la producción de proteína $C$ reactiva por los hepatocitos, lo que contribuye al síndrome inflamatorio ${ }^{6}$.
La variante ECM HHV8+ tiene un comportamiento clínico agresivo, se presenta frecuentemente con manifestaciones sistémicas, conlleva una mortalidad informada del $50 \%$ a 2 años y se considera una condición premaligna. Se ha informado transformación hasta en el $25 \%$ de los casos a LDCG-B, linfomas angioinmunoblásticos, linfomas plasmablásticos, linfomas de Hodgkin clásicos, sarcoma de Kaposi y plasmocitoma $^{6,24,38}$. Los pacientes con ECM HHV-8+ y $\mathrm{VIH}+$ tienen 15 veces más frecuencia de presentar un linfoma que la población $\mathrm{VIH}+\sin \mathrm{EC}^{20,33,38}$.

Algunos pacientes presentan cambios histopatológicos y hallazgos clínicos similares a los encontrados en la ECM HHV-8+, pero sin presentar la infección por HHV-8 ni por VIH; estos casos se conocen como ECM idiopática $(\mathrm{ECMi})^{20,40}$. Mientras que la ECM HHV-8+ es principalmente un trastorno linfoproliferativo con riesgo de progresión a linfoma, la ECMi permanece en controversia y posiblemente constituya más de una enfermedad. Datos recientes han delineado por lo menos un síndrome distintivo que se caracteriza por trombocitopenia, anasarca, fiebre, fibrosis reticulínica y organomegalia, referido por el acrónico TAFRO o también como enfermedad de Castleman-Kojima (vide infra). Este es un estado proinflamatorio hipercitocinémico similar a su contraparte, pero de origen desconocido ${ }^{20}$.

La enfermedad de Castleman-Kojima o síndrome TAFRO ocurre en adultos mayores, con edad media de 56 años, con una predilección en mujeres (4:1). No está asociado a infección viral por HHV-8, VIH ni VEB, e histológicamente es similar, pero tiene menor grado de plasmocitosis y una marcada proliferación vascular en el área interfolicular con centros germinales atróficos y ligeramente hialinizados. Las biopsias de médula ósea de estos pacientes generalmente tienen hiperplasia de megacariocitos, con emperipolesis asociada y ligera mielofibrosis. Estas características clinicopatológicas sugieren que el síndrome TAFRO puede representar un subtipo diferente al de la ECMi ${ }^{20,40}$.

El diagnóstico de la ECMi es más difícil, debido a que sus síntomas son poco definidos. Existen diferentes teorías acerca de su patogénesis, como anormalidades citogenéticas o un polimorfismo genético que involucra los genes del receptor de la IL-6, lo que sugiere una aberración en la línea germinal de los genes implicados en la regulación del sistema inmunitario innato ${ }^{41}$. Histológicamente muestra tanto características de la variante HV como de la $\mathrm{CP}^{20}$, con proliferación de CP policlonales en el área interfolicular y centros germinales relativamente normales. 
En resumen, la EC es un reto diagnóstico porque: 1) los hallazgos patológicos con hematoxilina y eosina no son del todo específicos, ya que pueden ser vistos en otras condiciones reactivas y neoplásicas; 2) su presentación clínica es heterogénea; y 3) puede estar asociada a virus, como VIH, HHV-8 y VEB, que pueden simular otros trastornos linfoproliferativos.

En la EC existe un aumento del número de CRF que expresan desmina y citoqueratina OSCAR, y hay positividad al EGFR en las CDF de los ganglios linfáticos afectados por la EC (en cualquiera de sus variantes, HV o CP), cuyo significado fisiopatológico se desconoce.

La presencia de CDF displásicas en los centros germinales atróficos de la EC se ha demostrado tanto por estudios citológicos como por estudios moleculares, lo que sugiere que una alteración genética en estas células puede ser la causa de la EC. La expresión del EGFR que caracteriza a las CDF presentes en la EC no se observa en otros tipos de hiperplasia folicular ni en linfomas, por lo que el EGFR positivo en las CDF podría utilizarse para establecer el diagnóstico de EC. Además, se ha planteado la posibilidad de que, al resultar positivo el EGFR, los agentes anti-EGFR puedan ser de utilidad como adyuvantes en regímenes sistémicos en casos de EC irresecables ${ }^{8,11}$.

\section{Bibliografía}

1. Casper C. The aetiology and management of Castleman disease at 50 years: translating pathophysiology to patient care. $\mathrm{Br} \mathrm{J}$ Haematol. 2005;129:3-17.

2. Cronin DMP, Warnke RA. Castleman disease. An update on classification and the spectrum of associated lesions. Adv Anat Pathol.2009;16:236-46.

3. Soumerai JD, Sohani AR, Abramson JS. Diagnosis and management of Castleman disease. Cancer Control. 2014;21:266-78.

4. Myloma EE, Baraboutis IG, Lekakis LJ, et al. Multicentric Castleman's disease in HIV infection: a systematic review of the literature. AIDS Rev. 2008;10:25-35.

5. Kojima M, Nakamura N, Tsukamoto $\mathrm{N}$, et al. Clinical implications of idiopathic multicentric Castleman disease among Japanese: a report of 28 cases. Int J Surg Pathol. 2008;16:391-8.

6. Uldrick TS, Polizzotto MN, Yarchoan R. Recent advances in Kaposi sarcoma herpesvirus- associated multicentric Castleman diseases. Curr Opin Oncol. 2012;24:495-505

7. Ye B, Gao S-G, Li W, Yang L-H, Zhao S-H, Zhu X-L, et al. A retrospective study of unicentric and multicentric Castleman's disease: a report of 52 patients. Med Oncol. 2010;27:1171-8.

8. Sun X, Chang K-C, Abruzzo LV, et al. Epidermal growth factor receptor expression in follicular dendritic cells: a shared feature of follicular dendritic cell sarcoma and Castleman's disease. Hum Pathol. 2003;34:835-40.

9. Chan JKC, Tsang WYW. Follicular dendritic cell tumor and vascular neoplasm complicating hyaline-vascular Castleman's Disease. Am J Surg Pathol. 1994;18:517-25.

10. Danon AD, Krishnan J, Frizzera G. Morpho-immunophenotypic diversity of Castleman's disease, hyaline-vascular type: with emphasis on a stroma-rich variant and a new pathogenetic hypothesis. Virchows Archiv A Pathol Anat Histopathol. 1993;423:369-82.

11. Walsh-Jahnke R, Cui W, Zhang D. Late recurrence of Castleman's disease with mixed angiomyoid, histiocytic reticulum cell, follicular dendritic cell stroma-rich proliferations: a case report and review of the literature. J Hematopathol. 2015;8:43-7.
12. Fletcher AL, Acton SE, Knoblich K. Lymph node fibroblastic reticular cells in health and disease. Nat Rev Immunol. 2015;15:350-61.

13. Bösmüller H, Klenske J, Bonzheim I, et al. Cytokeratin-positive interstitial reticulum cell tumor: recognition of a potential "in situ" pattern. Hum Pathol. 2016;49:15-21.

14. Tung KS, McCormack LJ. Angiomatous lymphoid hamartoma. Report of five cases of literature. Cancer. 1967;20:525-36.

15. Castleman B, Towne VW. Case records of the Massachusetts General Hospital weekly clinicopathological exercises: case 40011. N Engl J Med. 1954;250:26-30.

16. Castleman B, Iverson L, Menendez VP. Localized mediastinal lymph-node hyperplasia resembling thymoma. Cancer. 1956;9:822-30.

17. Festen C, Flendrig JA, Schilling PH. Giant lymphomas. Ned Tidschr Genneskd. 1969;113:1918-9.

18. Keller AR, Hochholzer L, Castleman B. Hyaline-vascular and plasma cell types of giant Lymph node hyperplasia of the mediastinum and other locations. Cancer. 1972;29:670-83.

19. Frizzera G, Massarelli G, Banks PM, et al. A systemic lymphoproliferative disorder with morphologic features of Castleman's disease. Pathological findings in 15 patients. Am J Surg Pathol. 1983;7:211-31.

20. Fajgenbaum DC, Uldrick TS, Bagg A, et al. International, evidence-based consensus diagnostic criteria for HHV-8-negative/idiopathic multicentric Castleman disease. Blood. 2017;29:1646-57.

21. Soulier J, Grollet L, Oksenhendler E, et al. Kaposi's sarcoma-associated herpesvirus-like DNA sequences in multicentric Castleman's disease. Blood. 1995;86:1276-80.

22. Bonekamp D, Horton KM, Hruban $\mathrm{RH}$, et al. Castleman disease: the great mimic. RadioGraphics. 2011;31:1793-807.

23. Schulte K-M, Talat N. Castleman's disease - a two compartment model of HHV-8 infection. Nat Rev Clin Oncol. 2010;7:533-43.

24. Ioachim HL, Medeiros LJ. Lymph node pathology. $4^{\text {th }}$ ed. Philadelphia: Lippincott William \& Wilkins; 2009. p. 227-37.

25. Kojima M. Shimizu K, Ikota H, et al. "Follicular variant" of hyaline-vascular type of Castleman's disease: histopathological and immunohistochemical study of 11 cases. J Clin Exp Hematopathol. 2008;48:39-45.

26. Pauwels $P$, Dal Cin P, Vlasveld LT, et al. A chromosomal abnormality in hyaline vascular Castleman's disease: evidence for clonal proliferation of dysplastic stromal cells. Am J Surg Pathol. 2000;24:882-8.

27. Cokelaere K, Debiec-Rychter M, De Wolf-Peeters $C$, et al. Hyaline vascular Castleman's disease with HMGIC rearrangement in follicular dendritic cells: molecular evidence of mesenchymal tumorigenesis. Am J Surg Pathol. 2002;26:662-9.

28. Chang K-C, Wang Y-C, Hung L-Y, et al. Monoclonality and cytogenetic abnormalities in hyaline vascular Castleman disease. Mod Pathol. 2014;27:823-31.

29. Franke WW, Moll R. Cytoskeletal components of lymphoid organs. I. Synthesis of cytokeratins 8 and 18 and desmin in subpopulations of extrafollicular reticulum cells of human lymph nodes, tonsils, and spleen. Differentiation.1987;36;145-63.

30. Coggi G, Dell'Orto $P$, Braidotti $P$, et al. Coexpression of intermediate filaments in normal and neoplastic human tissues. Ultrastruct Pathol. 1989;13:501-14

31. Schuerfeld K, Lazzi S, De Santi MM, et al. Cytokeratin-positive interstitial cell neoplasm: a case report and classification issues. Histopathology. 2003;43:491-4.

32. Cho J, Gong G, Choe G, et al. Extrafollicular reticulum cells in pathologic lymph nodes. J Korean Med Sci. 1994:9:9-15.

33. Van Rhee F, Stone K, Szmania S, et al. Castleman disease in the 21st century: an update on diagnosis, assessment, and therapy. Clin Adv Hematol Oncol. 2010;8:486-98.

34. Fedoriw $Y$, Dogan A. The expanding spectrum of follicular lymphoma. Surg Pathol Clin. 2016;9:29-40.

35. Jares $\mathrm{P}$, Colomer D, Campo E. Molecular pathogenesis of mantle cell Iymphoma. J Clin Invest. 2012;122:3416-23.

36. Talat N, Schulte K-M. Castleman's disease: systematic analysis of 416 patients from the literature. Oncologist. 2011;16:1316-24.

37. Chang $Y$, Cesarman E, Pessin MS, et al. Identification of herpesvirus-like DNA sequences in AIDS-associated Kaposi's sarcoma. Science. 1994;266:1865-9.

38. Dupin N, Diss TL, Kellam P, et al. HHV-8 is associated with a plasmablastic variant of Castleman disease that is linked to HHV-8-positive plasmablastic lymphoma. Blood. 2000;95:1406-12.

39. Gaba AR, Stein RS, Sweet DL, et al. Multicentric giant lymph node hyperplasia. Am J Clin Pathol. 1978;69:86-90.

40. Fajgenbaum DC, van Rhee F, Nabel CS. HHV-8-negative, idiopathic multicentric Castleman disease: novel insights into biology, pathogenesis, and therapy. Blood. 2014;123:2924-33.

41. Liu AY, Nabel CS, Finkelman BS, et al. Idiopathic multicentric Castleman's disease: a systematic literature review. Lancet Haematol. 2016; 3:e163-75. 